\title{
Comprehensibility of Texts, Level of the Class, Achievement in Mathematics and Turkish Lessons
}

\author{
G. Füsun ALACAPINAR*
}

\begin{abstract}
There are a number of people putting forward the viewpoint that there is a significant relationship between the comprehensibility of texts and the student's level of achievement in Turkish and Mathematics lessons. It is because of this fact that texts are to be appropriate to the level of the students. At this point, many learning-teaching principles have been proposed. Moreover, the text should be in line with the rules relating to inference, abstraction, level, schema, material organization, exercise and visual system in that both the comprehensibility and pedagogically of the text may be based on these rules to some extent. Apart from these, there is a mathematical model both designed and presented by Sönmez (2003) relevant to the comprehensibility of texts. The question of the research is the mathematical way suggested by Sönmez (2003) effective in determining the comprehensibility of the texts from the third year to the eight year at primary schools?
\end{abstract}

Key Words: Comprehensibility of texts, level of class, success.

\footnotetext{
*Dr., Kavaklıdere Elementary School, Classroom Teacher, fusunmireli@yahoo.com
} 


\section{SUMMARY}

Problem situation: There are a number of people putting forward the viewpoint that there is a significant relationship between the comprehensibility of texts and the student's level of achievement in Turkish and Mathematics lessons. It is because of this fact that texts are to be appropriate to the level of the students. At this juncture, many learning-teaching principles have been proposed. Moreover, the text should be in line with the rules relating to inference abstraction, level, schema, material organization, exercise and visual system in that both the comprehensibility and pedagogicality of the text may be based on these rules to some extent. Apart from these, there is a mathematical model both designed and presented by Sönmez relevant to the comprehensibility of texts. In this research, the validity and reliability of this model was tested. That the model was consistent was found by the researcher.

The Aim of the Research and its Questions: Is the mathematical way suggested by Sönmez effective in determining the comprehensibility of the texts from the third year to the eight year at primary school?

1. There is not a significant relationship between students' Turkish lesson final exam grades and their average scores obtained from the text presented without codes.

2. There is not a significant relationship between students' Mathematics lesson final exam grades and their average scores obtained from the text presented without codes.

3. There is not a significant relationship between the level of the class and the comprehensibility of the text.

Method: In this research, the procedure followed is as follows: The text developed by Sönmez was utilized in its original form. All the words in this text were coded. After that, all the words first with codes $\% 99, \% 90, \% 80 ; \% 70 ; \% 60$; $\% 50, \% 40, \% 30 ; \% 20, \% 10, \% 01$ and finally without codes were given to the students in the same session in two hours time.

Findings: There was not found a significant relationship between the comprehensibility of the texts and mean success scores of the class, between the comprehensibility of the texts and mean success scores in Mathematics lesson and between the comprehensibility of the texts and mean success scores in Turkish lesson.

Suggestions: The mathematical model suggested by Sönmez may be utilized in the educational setting. Experimental researches may be done on a wider sampling. 


\title{
Metinlerin Anlaşırlığı ile Sınıf Düzeyi, Matematik ve Türkçe Derslerindeki Başarı
}

\author{
G. Füsun ALACAPINAR
}

ÖZ. İlköğretim Okulu 3. sinıftan 8. sınıfa kadar metinlerin anlaşılabilir olmasını saptamada Sönmez tarafından önerilen matematiksel yol etkili midir?

1.Öğrencilerin Türkçe yıl sonu başarı notu ile şifresiz olarak sunulan metinden aldıkları puan ortalamaları arasında anlamlı bir ilişki yoktur.

2.Öğrencilerin matematik yıl sonu başarı notu ile şifresiz olarak sunulan metinden aldıkları puan ortalamaları arasında anlamlı bir ilişki yoktur.

3. Sınıf düzeyiyle metnin anlaşılırlığı arasında anlamlı bir ilişki yoktur.

Metinlerin anlașılırlığı ile sınıf, matematik ve Türkçe dersindeki başarı ortalamaları arasında anlamlı bir ilişki bulunmamıştır. Eğitim ortamında Sönmezin önerdiği matematiksel model kullanılabilir. Daha geniş bir örneklem üzerinde deneysel araştırmalar yapılabilir.

Anahtar Sözcükler: Metinlerin anlaşılırlığı, sınıf düzeyi, başarı

\section{GİRIŞ}

Metinlerin anlaşılır olması eğitimin önemli ilkelerinden biridir. $\mathrm{Bu}$ konuda araştırmalar yapılmış ve pek çok ilke ileri sürülmüştür (Sönmez 2003; Anderson 1992; Berry 1996; Annis \& Ferril 1985; Brdomage \&

* Dr., Kavaklıdere İlköğretim Okulu Sınıf Öğretmeni, fusunmireli@yahoo.com 
Mayer 1981; Caeur 1997; Duchastel\& Hungester 1984; Dumortuier 1995; Labesse 1999; Leitner 1990, Miles 1990; Laubach Literacy of Canada 2005; University of Minnesota 1998). Ayrıca metinlerin anlaşırlığını belirlemede ilk çalışmalar ABD'de başlamış ve Fog İndexi adıyla Robert Gunning tarafindan 1952 yllında matematiksel bir formül geliştirilmiştir. $\mathrm{Bu}$ matematik formüle göre metinlerin anlaş1lırlı̆̆ını hesaplamada şöyle bir yol izlenmektedir:

1.Metindeki sözcüklerin sayımı. 2. Metindeki cümlelerin sayımı. 3. Üç ve daha fazla heceden oluşan sözcüklerin sayımı. 4. Sözcük sayısının cümle sayısına bölünmesi. 5. Çıkan sonuca, metindeki üç ve daha fazla olan sözcük sayısının eklenmesi. 6. Sonucun 0.4 ile çarpılması (Sönmez 2003, Budak 2005). Fog İndeks'inin 7-8 olması metnin anlaşılırlığının ideal; 12 ve daha yüksek olması ise metnin anlaşılmasının zor olduğunu gösterir (University of Minnesota 1998).

Sönmez tarafından yapılan araştırma, Gunning modelinin işlemediğini göstermektedir. En azından Türkçe için uygun değildir. Sönmez'in geliştirip önerdiği matematiksel formül ise şu basamaklardan oluşmaktadır:

1.Metindeki sözcük sayısının cümle sayısına bölünerek sözcük oranının bulunması. 2. Metindeki anlamı bilinmeyen sözcük, deyim, terim, kavram, mecaz, benzetme, formül, sembol sayısının toplam sözcük sayısına bölünerek güçlük oranının belirlenmesi. 3. Metindeki anlamı bilinmeyen sözcük, deyim, terim, kavram, mecaz, benzetme, formül, sembol sayısının metindeki cümle sayısına bölünerek anlam oranının belirlenmesi. 4. Anlam oranının sözcük oranına bölünüp güçlük oranıyla çarpılıp anlaşılırlık oranının bulunması. Daha kısa bir yolla güçlük oranının karesi alınarak anlaşılırlık oranı bulunabilir; çünkü bu da aynı sonucu verir ( Sönmez 2003).

$\mathrm{Bu}$ iki çalışmanın dışında metnin anlaşılır olması; kişinin metnin örüntüsünde yer alan öğelere ve bunlara anlam kazandıran bağlamsal yapıya hâkimiyet düzeyine, konuyla ilgisine, okuma becerisindeki düzeyine bağ $1_{1}$ olabilir (Budak 2005). Yapılan araştırmaların büyük bir çoğunluğu Gunning'in Fog İndeks'inin geçerli ve güvenilir bir yol olmadığını destekler niteliktedir. Buna karşılık Budak'ın da belirttiği gibi Sönmezin önerdiği matematiksel modelin en azından Türkçe için güvenilir sonuçlar verdiği söylenebilir (Budak 2005).

Budak'ın araştırmasında vurguladığı gibi metinlerin anlaşılırlığını saptamada pek çok yol olabilir. $\mathrm{Bu}$ iş matematiksel bir modele de indirgenemeyebilir (Budak 2005). Bu araştırmanın amacı, metinlerin anlaşılır olmasıyla, öğrencilerin Türkçe ve matematik başarı ortalamaları ve sınıf düzeyleri arasında anlamlı bir ilişkinin olup olmadığını saptamaktır. 


\section{Problem Cümlesi}

Ankara'da ......... İlköğretim Okulu 3. sinıftan 8. sinıfa kadar metinlerin anlaşılabilir olmasını saptamada Sönmez'in önerdiği matematiksel model etkili midir?

\section{Denenceler}

1.Öğrencilerin Türkçe yıl sonu başarı notu ile şifresiz olarak sunulan metinden aldıkları puan ortalamaları arasında anlamlı bir ilişki yoktur.

2.Öğrencilerin matematik yıl sonu başarı notu ile şifresiz olarak sunulan metinden aldıkları puan ortalamaları arasında anlamlı bir ilişki yoktur.

3. Sınıf düzeyiyle metnin anlaşılırlığı arasında anlamlı bir ilişki yoktur.

\section{YÖNTEM}

$\mathrm{Bu}$ araştırmada şöyle bir işlem sırası izlendi: Sönmez tarafından geliştirilen metin aynen kullanıldı. Bu metindeki sözcüklerin tümü şifrelendi. Sonra sirasiyla $\% 99, \% 90, \% 80 ; \% 70 ; \% 60 ; \% 50, \% 40, \% 30 ; \% 20, \% 10$, $\% 01$ şifreli ve sonunda tümü şifresiz olarak aynı oturumda iki saatlik süre içinde verildi. Ankara'da ........... İlköğretim Okulu 3-8 sinıflar içinden sistematik random yoluyla onar öğrenci saptandı.

\section{BULGULAR}

$\mathrm{Bu}$ başlık altında toplanan veriler önce tablolaştırıldı. Sonra uygun istatistik teknikler kullanılarak analiz edildi. Yorumlanıp bir sonuca varıldı.

Metnin Tümü Şifreli ve Sinıfların Toplam Ortalama Puanları

Metnin tümü şifreli halde verildiğinde sınıfların toplam ortalama puanları Tablo 1'de sunuldu.

Tablo 1. Metnin Tümü Şifreli

\begin{tabular}{lll}
\hline Sinıflar & Öğrenci sayısı & Ortalama \\
\hline 3.sinıf & 10 & 0 \\
$4 . \sin ı f$ & 10 & 0 \\
5.sinıf & 10 & 0 \\
6.sinıf & 10 & 0 \\
7.sinıf & 10 & 0 \\
8.sinıf & 10 & 0 \\
\hline
\end{tabular}

Tablo 1'de görüldüğü gibi tüm sinıfların metnin tümü şifreli olunca aldıkları puanların ortalaması sıfırdır. Hiçbir öğrenci metni anlayamamıştır. Hatta şaşırıp kalmışlar, kâğıtlarını ortalama 27 saniye içinde geri getirmişlerdir. Araştırmacının yüzüne garip garip bakmışlar " $\mathrm{Bu}$ nedir öğretmenim? Anlayamadım. Ne yapacaktık?” gibi ifadeler kullanmışlardır. 
Budak ve Sönmez'in de yaptığı araştırmalarda metindeki tüm sözcükler anlamsız yani şifreli olunca, öğrenciler aynı tepkiyi göstermişlerdir (Sönmez 2003; Budak 2005). ( 3. Denenceyle ilgili)

Metnin Tümü Ana Dille Şifresiz ve Siniflartn Toplam Ortalama Puanlart

Metnin tümü anadille ve şifresiz halde verildiğinde sinıfların toplam ortalama puanları Tablo 2'de sunuldu.

Tablo 2 . Metnin Tümü Anadille Şifresiz verildiğinde Toplam Ortalama Puan

\begin{tabular}{llll}
\hline Sinıflar & Öğrenci Sayısı & Genel Ortalama & S \\
\hline 3.sınıf & 10 & 8.7 & .94 \\
4.sınıf & 10 & 8.4 & .51 \\
5.sinıf & 10 & 8.5 & .70 \\
6.sınıf & 10 & 8.6 & .51 \\
7.sınıf & 10 & 8.5 & .84 \\
$8 . \sin ı f$ & 10 & 8.3 & .67 \\
\hline
\end{tabular}

Tabloda görüldüğü gibi 3.sinıfların toplam ortalama puanı 8.7; dördüncü sınıfların 8.4; beşinci ve yedinci sınıfların 8.5; altıncı sınıfların 8.6; sekizinci sınıfların ise 8.3'tür. Sınıfların toplam puan ortalamaları arasında anlamlı bir farkın olup olmadığı çok yönlü varyans analiziyle yapılmış ve .05 manidarlık ve çeşitli serbestlik derecesiyle $F$ değeri anlamlı bulunmamıştır. $\mathrm{Bu}$ verilere dayanarak sınıf düzeyiyle metnin anlaşılırlık oranı arasında anlamlı bir farkın olmadığı söylenebilir. Yani sınıf düzeyi değişince, metnin anlaşılırlık düzeyi değişmemektedir. Diğer bir değişle sınıf düzeyiyle metnin anlaşı1ırlık düzeyi arasında anlamlı bir ilişkinin bulunmadığ 1 söylenebilir. Budak ve Sönmez'in araştırmalarında metnin tümü anlaşılır olunca, öğrenciler soruları yanıtlayabilmişler ve bu sonuç sınıf düzeyi değişince anlamlı derecede değişmemiştir (Sönmez 2003; Budak 2005).

\section{Metnin Şifrelenme Oranlariyla Anlama Düzeyleri}

Metnin şifrelenme oranına göre sınıfların aldıkları puan ortalamalarıyla ilgili veriler Tablo 3 'te sunulmuştur. 
Tablo 3. Sinıfların Metnin Şifrelenme Oranına Göre Aldıkları Notların Ortalamaları (10 tam not üzerinden )

\begin{tabular}{lccccccccccc}
\hline Sinıflar & $\% 99$ & $\% 90$ & $\% 80$ & $\% 70$ & $\% 60$ & $\% 50$ & $\% 40$ & $\% 30$ & $\% 20$ & $\% 10$ & $\% 01$ \\
& Şifreli & şifreli & şifreli & şifreli & şifreli & şifreli & şifreli & şifreli & şifreli & şifreli & şifreli \\
\hline 3. sinıf & 0 & 0 & 0 & 0 & 0 & 0.8 & 1.0 & 0.7 & 2.7 & 6.4 & 7.2 \\
4.sinıf & 0 & 0 & 0 & 0.25 & 0.7 & 1.1 & 1.2 & 1.8 & 3.3 & 5.9 & 7.3 \\
5.sinıf & 0 & 0 & 0 & 0.5 & 0.8 & 1.2 & 1.5 & 1.7 & 3.1 & 5.7 & 7.1 \\
6.sinıf & 0 & 0.04 & 0.04 & 0.4 & 0.8 & 1.2 & 1.4 & 2.3 & 5.9 & 6.2 & 7.2 \\
7.sinıf & 0 & 0 & 0 & 0 & 0.6 & 1.0 & 1.0 & 1.5 & 3.0 & 7.0 & 7.6 \\
8.sinıf & 0 & 0 & 0 & 0 & 0.6 & 0.8 & 0.9 & 1.1 & 2.9 & 6.8 & 7.5 \\
\hline
\end{tabular}

Tablo 3'te görüldügü gibi metnin şifreleme düzeyi \%20'den sonra, onu anlama düzeyi tüm sınıflarda yükselmektedir. Yapılan çok yönlü varyans analizi sonuçlarına göre sınıfların puan ortalamaları arasında anlamlı bir fark bulunmamıştır. Bu verilere dayanarak bilinen sözcük sayısı artıkça, metni anlama düzeyi tüm sınıf düzeylerinde yükselmektedir. Dikkat edilirse, metnin şifrelenme düzeyi $\% 20$ 'den daha düşük olunca, onun tüm sınıflarda anlaşılır olma düzeyinin yükseldiği söylenebilir. Metindeki şifreleme düzeyi yükselince, özellikle \%60'dan \%99'a doğru gidildikçe, tüm sinıflardaki öğrencilerin metni anlama puan ortalamaları sıfıra düşmektedir. Bu verilere dayanarak metnin anlaşılırlığının tüm sınıflarda metnin şifreleme düzeyine göre (\%99'a doğru gidildikçe) düştüğü ve (\%01'e doğru gidildikçe) yükseldiği söylenebilir. Yani metnin anlaşılırlığının sınıf düzeyiyle değil, anlaş1lırlık düzeyiyle ilgili olduğu savunulabilir. $\mathrm{Bu}$ veriler Budak ve Sönmezin de belirttiği gibi metinde bilinen sözcük sayısı artıkça anlama düzeyi yükselmekte, azaldıkça metni anlama düzeyi düşmektedir (Budak 2005; Sönmez 2003).

Sınıfların Türkçe Dersinden Aldıkları Ağırlıklı Yıl Sonu Ortalamaları Ile Yüzde Yüz Şifresiz Metinden Aldıkları Puanlar

Sınıfların Türkçe dersinden aldıkları ağırlıklı yıl sonu ortalamaları ve yüzde yüz şifresiz verilmiş metinden aldıkları ortalama puanlarla ilgili veriler Tablo 4'de sunulmuşstur. 
Tablo 4. Sinıfların Türkçe Dersinden Aldıkları Ağırlıklı Yıl Sonu Ortalamaları (tam not 10 üzerinden) ve Şifresiz Anadille Verilmiş Metinden Aldikları Ortalama Puanlar

\begin{tabular}{ccc}
\hline Sinıflar & $\begin{array}{c}\text { Türkçe } \\
\text { x }\end{array}$ & $\begin{array}{c}\text { \%100 Şifresiz } \\
\text { X }\end{array}$ \\
\hline 3. sinıf & 9.3 & 8.7 \\
4.sinif & 9.4 & 8.4 \\
5.sinif & 9.3 & 8.5 \\
6.sinif & 7.4 & 8.6 \\
7.sinıf & 7.0 & 8.5 \\
8.sinıf & 7.7 & 8.3 \\
\hline
\end{tabular}

Sınıfların Türkçe dersinden aldıkları ağırlıklı yıl sonu ortalamaları ve yüzde yüz şifresiz verilmiş metinden aldıkları ortalama puanlar arasında anlamlı bir ilişkinin olup olmadığı Pearson Momentler Çarpımı Korelasyon Katsayısı (r) ile hesaplanmıştır. Bulunan 0.15 değeri, 05 manidarlık ve beş serbestlik derecesiyle anlamsız bulunmuştur. Bu verilere dayanarak Türkçe dersinden aldıkları yıl sonu ağırlıklı puan ortalamalarıyla, metnin tümünün şifresiz olması arasında anlamlı bir ilişkinin olmadığı söylenebilir. Metnin anlaşılırlığının Türkçe dersinden alınan notla ilişkili olmadığı savunulabilir. Ayrıca sınıf düzeyiyle de metin anlaşı1ırlık düzeyi arasında da anlamlı bir ilişki bulunmamıştır. $\mathrm{Bu}$ iki sonuç Budak'ın verilerini desteklemektedir. Budak da öğrencilerin Fransızca dönem sonu notlarıly metnin tümünün şifresiz olarak verildiğinde aldıkları notların ortalamaları arasında anlamlı bir ilişki bulmamıştır ( Budak 2005).

Sinıfların Matematik Dersinden Aldıkları Ağırlıklı Yıl Sonu Ortalamaları Ille Yüzde Yüz Şifresiz Metinden Aldıkları Puanlar

Sınıfların matematik dersinden aldıkları ağırlıklı yıl sonu ortalamaları ve yüzde yüz şifresiz anadille verilmiş metinden aldıkları ortalama puanlarla ilgili veriler tablo 5'te sunulmuştur.

Tablo 5. Sinıfların Matematik Dersinden Aldıkları Ağırlıklı Yıl Sonu Ortalamaları (tam not 10 üzerinden) ve Şifresiz Anadille Verilmiş Metinden Aldikları Ortalama Puanlar

\begin{tabular}{lll}
\hline \multirow{2}{*}{ Sinıflar } & Matematik & $\begin{array}{l}\text { \%100 Şifresiz } \\
\mathrm{x}\end{array}$ \\
\hline 3. sinıf & 8.7 & 8.7 \\
4.sinif & 9.0 & 8.4 \\
5.sinif & 8.9 & 8.5 \\
6.sinıf & 6.5 & 8.6 \\
7.sinif & 4.7 & 8.5 \\
8.sinif & 5.2 & 8.3 \\
\hline
\end{tabular}


Sinıfların matematik dersinden aldıkları ağırlıklı yıl sonu ortalamaları ve yüzde yüz şifresiz anadille verilmiş metinden aldıkları ortalama puanlar arasında anlamlı bir ilişkinin olup olmadığı Pearson Momentler Çarpımı Korelasyon Katsayısı (r) ile hesaplanmıştır. Bulunan 0.32 değeri 05 manidarlık ve beş serbestlik derecesiyle anlamsız bulunmuştur. Bu verilere dayanarak matematik dersinden aldıkları yılsonu ağırlıklı puan ortalamalarıyla, metnin tümünün şifresiz olması arasında anlamlı bir ilişkinin olmadığı söylenebilir. Metnin anlaşılırlığının matematik dersinden alınan notla ilişkili olmadığı savunulabilir. Ayrıca sınıf düzeyiyle de metin anlaşılırlık düzeyi arasında da anlamlı bir ilişki bulunmamıştır.

Araştırmaya katılan her sınıftan random yoluyla seçilen altı öğrenciyle yapılan odak grup görüşmesinde şu görüşler ileri sürülmüştür: (Öğrencilerin bazıları görüşlerini ifade ederken cümle bozukluğu yaptığından, bunlar, onların onayı da alınarak anlamı bozmayacak şekilde düzeltilerek sunulmuştur):

K.1. Önce metnin ne olduğunu anlayamadım. Bana Çince gibi geldi. Sonra tüm metin gelince anladım.

K.2. Ne yapmak istediğinizi anlayamadığım için korktum. Türkçe olunca parçayı anlayıp yanıtladım. Bu araştırma hoşuma gitti.

K.3. Önce zayıf alacağım dedim. En son verdiğiniz okuma hoşuma gitti. Her soruya cevap verdim.

K.4. Öğretmenin niçin böyle bir iş yaptığınızı önce anlamadım. Metinlerde Türkçe artınca sevindim. Yapınca çok güzel oldu.

K.5. Önce bizi niçin şaşırttınız? Anlayamadım. Anlayacağım şekilde parça gelince çok sevindim. Ne yapmak istediğinizi sonra çok iyi anladım. Herhalde kelimeleri bilince ve bilmeyince ne yapacağımızı denediniz, değil mi öğretmenim?....

K.6. Başta, bu iş bana çok saçma geldi. Şaşırdım... Korktum... Son yedi sınava kadar ne olduğunu anlayamadım. Yedi metinden sonra anladım. Hoşuma gitti. Demek kelimeleri bilmeyince parçayı anlayamıyorum. Oysa parça ne kadar kolaymış...

Yapılan odak grup görüşmesinde, öğrenciler, tüm metin şifreli verildiği zaman onu anlayamamışlar ve bundan dolayı da korku, tedirginlik duymuşlar ve şaşırmışlardır. $\mathrm{Bu}$ doğal bir durumdur. Kişi anlamadığı şeylerle ilgili, bu duygulara kapılabilir. Öğrencinin korkusu zayıf alacağından da kaynaklanabilir. Nitekim bir öğrenci "önce zayıf alacağım." diyerek bu korkusunun nedenini belirtmiştir. Korku, anlamayı yani iletişimi 
güçleştirebilir; engelleyebilir; çünkü o bir bakıma ceza olarak iş görür. Cezanın öğrenmeyi güçleştirdiği bilinmektedir (Senemoğlu 1997). Bu durum, doğru iletişim kurmayı da engeller. Nitekim öğrencilerin hepsi, önce metni anlamadıklarını vurgulamışlardır. Metni anlamamak, öğrencinin iletişim kuramamasının bir göstergesidir. İletişimin açık ve anlaşılır olmaması öğrenmeyi engeller. Tersi bir durum da ise; iletişim kolaylaşabilir ve metin anlaşılabilir; böylece öğrencinin çok yönlü gelişimi sağlanabilir (Ergin 1995; Erden ve Akman 1995; Gözütok 2006; Küçükahmet 1992; Gürkan 2001). Nitekim öğrenciler, önce metnin \%70'i ve giderek tümü şifresiz verildiğinde, onu anladıkları için sevinmişler, korku ve tedirginlikleri ortadan kalkmış; üstelik araştırmanın amacını bile belirleyebilmişlerdir. Ayrıca öğrenci yanıtlarında, "Bir metni anlayabilmenin, o metindeki sözcüklerin anlamını bilmekle ilgili olduğu" da vurgulanmıştır. Bu yargı, araştırmanın temel savını da desteklemektedir.

\section{TARTIŞMA VE SONUÇ}

Metinlerin anlaşılır olup olmaması öğrencinin bulunduğu sınıfa, Türkçe ve matematik derslerindeki başarılarına bağlı olmaması şöyle yorumlanabilir: Öğrenci için metnin anlaşılması, o metindeki sözcüklerin bilinmesiyle doğrudan ilişkili olabilir. Nitekim bir metinde anlamı bilinmeyen sözcüklerin sayısı artıkça, metnin anlaşılması güçlenmektedir. Bunun için kavram, terim öğretimi önemli bulunmuştur. Nitekim Bloom basamaklarında da öncelik terimler bilgisine verilmiştir. Bloom tarafından oluşturulan taksonomide bilgi basamağında kavramalar, olgular, alışılar, sınıflamalar, yöntem ve ilkeler bilgisine aşamalı olarak öncelik verilmesinin nedeni buna bağlanabilir. Öğrenci kavramları, olguları, alışıları (sembol ve formüller), sınıflamaları, yöntem ve ilkeleri bilmediği zaman kavrama ve daha üst düzeylerdeki basamaklara geçemiyor; çünkü metni anlayamıyor; ya da anlamakta güçlük çekiyor (Demirel 1996; Güneş 2000; Arslan 2007). Bu olgu, pek çok kez sınıfta ders işlerken gözlemlendi. Türkçe ve matematik derslerinin birinci basamağı metni, problemi anlamadır. Türkçe'de metni anlama, kavramların, terimlerin, yabancı sözcüklerin, değimlerin, mecazların, benzetmelerin ne anlama geldiğini bilme, cümle içinde doğru kullanma vb. davranışları içerir. Aynı şekilde matematik dersinde de verilenleri ve istenilenleri yazma, söyleme problemi anlama basamağının önkoşul davranışlarıdır. Problemi anlamadan öğrencinin onu çözmesi beklenemez. Nitekim odak grup görüşmesinde de öğrenciler bu yargıyı destekler nitelikte ifadelerde bulunmuşlardır. Yapılan araştırma bu ilkeyi desteklemektedir.

Diğer bir neden ise iletişimin doğasıyla ilgili olabilir. İletişim, iletinin uygun kanallarla alıcıya en yakın anlamıyla ulaştırılmasını içerir. İletinin 
taşıdığı anlamın aynı biçimde alıcıya iletilmesini etkileyen önemli etkenlerden biri de kanallar olabilir. Bu kanallar iletinin anlamını gölgeleyebilir, ya da açık ve anlaşılır hale getirebilir. Cümlelerin uzunluğu, kısalığı iletişimi zorlaştıran ya da kolaylaştıran nedenlerden biri olabilir.

Ayrıca eğitim sisteminde bilgi düzeyine ağırlık verilmiş, kavrama ve daha üst düzey becerilere gerekli önem verilmemişse, öğrencinin metni anlaması zorlaşabilir. Sönmez, Budak ve Alacapınar tarafından yapılan bu araştırmalar da öğrencilerin kestirme basamağına gelmedikleri gözlenmiştir. Kestirme basamağına gelen öğrenciler bazı sözcüklerden, hele \%50'si şifrelendiğinde yararlanarak metnin anlamını çıkarabilirlerdi. Ama hiçbir sınıfta bu iş başarılmamıştır. Bunun nedeni eğitim ortamında kestirme basamağına önem verilmemesine bağlanabilir.

\section{Öneriler}

Daha geniş bir grupla ve uzun sürede araştırmalar yapılabilir. Eğitim ortamında bilmecelere, bulmacalara yani kestirme basamağındaki davranışları geliştirici etkinliklere ağırlık verilebilir. Bunun için Türkçe'de cümlenin gelişinden bilinmeyen sözcüklerin anlamlarını bulma, çıkarma; belli yerleri kesilmiş metinleri sunarak bundan sonra ne olabilir gibi sorulara; geçmişi ve geleceği tahmin etme etkinliklerine, matematikte problemin sonucunu tahmin etmeye, çözümü için başka hangi verilerin gerektiğine yer verilebilir. Bu tür etkinliklerle öğrencinin kestirme gücü geliştirilebilir. Öğretmenlerin kestirme gücünü geliştirici öğrenme-öğretme etkinliklerinin nasıl kullanılacağı konusunda uygulamalı bir eğitimden geçirilmesi gerekebilir.

\section{KAYNAKÇA}

Anderson, L. F. (1992). Windows of the world the houghton social studies program. Boston: Allyn and Bacon A Division of Simon \& Schuster, Inc.

Annis, L., \& Ferril, C. (1985). Student generated paragraph summaries and the information-processing theory of proce learning. American Educational Research Association, 121, 153-172.

Arslan, C.(2007). Yazınsal Nitelikli Çocuk Kitaplarının Çocuğun Okuduğunu Anlama Ve Yazılı Anlatım Becerisine Etkisi. Eğitim Araştırmaları. Bahar. Say1:27. s.15-29.

Berry, A. (1996).Sadede gelelim. sonsuzluğun kıyıları, bilim dünyasından şaşırtıcı, ama gerçek öyküler. (Çev:A. Biçer). TÜBİTAK. Ankara.

Brdomage, B. K., \& Mayer, R. E. (1981). Relationship between what is remembered and creative problem solving performance in sentence learning. Journal of Educational Psychology, 72, 64-68. 
Budak, Y. (2005). Metinlerin Okunabilirlik Düzeyinin Saptanmasına Yönelik Eleştirel Bir Bakış Eğitim Araştırmaları , 21, 76-87

Coeur, F. (1997). Un état de recherches sur la lisibilité textuelle, Rapport de recherche bibliographique. Lyon: AGRAP Sciences Presse.

Demirel, M. (1996). "Bilgilendirici Metin Türünün Ve Okuduğunu Kavrama Becerisinin Altıncı Sınıf Öğrencilerinin Öğrenme Düzeyine Etkisi.” Yayımlanmamış doktora tezi, Hacettepe Üniversitesi, Sosyal Bilimler Enstitüsü, Ankara .

Duchastel, H., \& Hungester, S. (1984) Selected learning; an interpretive review of orienting factors in prose learning. Learning Processes. Learning Theories, 276, 24-30.

Dumortier, J. (1995). "Lisıbllte du discours didacdique - Réflexions sur la compréhension en lecture des différents écrits disciplinaires.» [Online] Retrieved on 10-March-2005, at URL: http://www.ulg.ac.be/cifen/

Erden, M ve Akman Y.(1995). Eğitim Psikolojisi. Arkadaş Yayınevi. Ankara.

Ergin, A. (1995). Öğretim Teknolojisi İletişim. PEGEM. Ankara.

Güneş, Firdevs. (2000). Okuma Yazma Öğretimi Ve Beyin Teknolojisi. Ocak

Yayınları.Ankara.

Gürkan, T. (2001). Bireyin Çok Yönlü Gelişimi. Bilim ve Aklın Aydınlığında Eğitim Dergisi. Aralı. Sayı:22.

Gözütok, D. (2006). Öğretim Illke Ve Yöntemleri. Ekinoks. Ankara.

Gunning, R. (1952). The technique of clear writing. (Çev: A. Biçer) New York: Ankara. (Özgün kitap 1952'de yayımlandı.)

Küçükahmet, L. (1992). Öğretim İlke Ve Yöntemleri. G.Ü. Yayınları. Ankara.

Labasse, B. (1999). La lisibilite redactionnelle: Fondements et perspectives. Communicatione \& langages. Paris : Armand Collin, 121, 86- 103

Laubach Literacy of Canada. (Kuruluş) (2005). "Overview of the Gununnig fog index" [Online]. Retrieved on 15-March-2005, at URL: http://www.laubachon.ca/Trainingpost/index.html

Leitner, G. (1990). Students uses of gramers of english-Can we avoid teaching. Irol, $112,155-167$.

Miles, T. H., (1990). Critical thinking and writing for science and technology. San Diego, CA: Harcourt Brace Jovanovich.

Senemoğlu, N. (1997). Gelişsim Öğrenme ve Öğretim - Kuramdan Uygulamaya Ankara: Spot Matbaacilık.

Sönmez, V. (2003). Metinlerin Eğitselliğini Saptamada Matematiksel Bir Yaklaşım Ĕ̈itim Araştırmaları. 10, 24-39.

University of Minnesota. (1998). "Policy and process Development Office, fog index"[Online]. Retrieved on 9-February-2006, at URL: http://www.fpd.finop.umn.edu. 


\section{EKLER}

\section{METINLER}

\section{ANADİLLE VERILEN METIN (Ek 1)}

Ali okuldan çıkınca, servise binip hemen eve geldi. Kapıyı anahtarıyla yavaşça açıp içeriye girdi. Çantasını omuzundan alıp vestiyere koydu. Paltosunu çıkarıp astı. Ayakkabılarını çıkarıp terliklerini giydi. Çantasını alıp koridoru hızla geçip odasına gitti.

Yarın için ödevleri vardı. Onları yapması gerekiyordu. Önce matematikle işe başladı. Çözülecek on problem vardı.Onlarla uğraştı. Beşinci soru kendisini biraz zorladı; ama onun da üstesinden geldi. Türkçe ödevi uzun zamanını almadı. Assıl işi seçtiği proje ile ilgiliydi. O çok zamanını alıyordu.İnternete giriyor, ilgili üniversite hocalarıyla ve uzmanlarla konuşuyor, kitaplar okuyor, sürekli not tutuyordu. Resim çekiyor, taslaklar çiziyor, planlar yapıyordu. Bu akşam da yine projeyle uğraşacaktı.

Aşağıdaki soruları bu metne göre yanıtlayınız:

S.1. Ali nereden geliyor?

S.2. Ali nereye gidiyor?

S.3. Ali’nin yarın hangi derslerden ödevi var?

S.4. Önce hangi ödevini yapıyor?

S.5. Hangi soruda zorlaniyor?

S.6. Sonra hangi ödevini yapıyor?

S.7. Bu akşam yine ne yapacak ?

S.8. Ali nasıl bir öğrencidir? Metinden yararlanarak söyleyiniz ?

S.9. Buradaki olayları üç cümleyle özetleyiniz.

S.10. Onun çoğu zamanını ne alıyor ? Bu çalışması için neler yapıyor?

\section{TÜMÜ ŞIFRELENMIŞ METIN (Ek 2)}

Xxx yyyyyy zzzzzzz vvvvvvv 11111 ddddd etf eeeee. Kkkkkk 111111111111 aaaaaa bbbb $\operatorname{ceccccc}$ ççççç. Çççççççççç omferhjke akmn vstjoyrt kyrtd. Pskyjsynf jjjjjjj mmmm. Nnnnnnnnnnnnnnn jjjjjjj öööööööööööö ppppp. Rrrrrrrrr gggg ssssssss şşşşş ttttt rrrrrrr wWwww.

$\begin{array}{llllllll}11111 & 2222 & 33333333 & 44444 . & 555555 & 6666666 & 77777777777 . & 8888\end{array}$ 99999999999 abc çdelade. 7654321ab a3 acdefghj 44444. 5555555 defghay. Kldefgh yzlk defghjmnp zhlmn nrkmrdn; nmn nyzn ry 12356azynm eeee. Nyzvsx 33333 bcde zındyazl aymçcı. Nkld 1lz snkmorp klmnv abc nrpsştavs. X çrk zmnınrpk aiijklmı. Aejmntsşö 4444444, mklnde untrpslmzx habtzlzztab xv ulkmlkgs 
kmnlm2346, ktaşlpmnr ozxwms, srkmnxy nsp taeirpzxy. Rslxy çynmlxz; tsnmnlkxz çzlmmnvk; pnmęden ypnkmxzsş. Bx aakmş nd ynxz klmnvsş uıtkşktlmxz.

Anklmdtsz yzlkntmx km mtknğ grj yntmlaiseixz:

1.Xxx nrdxyzf eeeeeee?

2. Xxx nrdxyf eeeee?

3. Xxxxxx 22222 huikm drlgkmnxy 33333444 ?

4. 8888 hujkm 3333333 ypmnxvz?

5. Hujkm yzlkon nrkmrdnikn?

6. Snmrı hujkm 3333333666666 ?

7. Bx aakms ynxz nx 6666666 ?

S.8. Xxx nsalı ibr örğendciıx? Mtindexz yxmnjklsgrty gedrfvbnyu?

S.9. Bgymnhdw olytrdxz lk crthjklş öcvfedsawyu.

S.10. Onut çklt zhjklşpı nk akjytr? Bn çskukmsdy iloy nhuyt yokjhgf?

\%99'U ŞIFRELENMIŞ METIN (Ek 3)

Xxx yyyyyy zzzzzzz vvvvvvv 11111 ddddd etf eeeee. Kkkkkk 111111111111 aaaaaa bbbb ccccccc ççççç. Ççççççççç omferhjke akmn vstjoyrt kyrtd. Pskyjsynf jjjjjjj mmmm. Nnnnnnnnnnnnnn jjjjjjj öööööööööööö ppppp. Rrrrrrrrr gggg ssssssss şşşş ttttt rrrrrrr wwwww.

$\begin{array}{llllllll}11111 & 2222 & 333333333 & 44444 . & 555555 & 6666666 & 77777777777 . & 8888\end{array}$ 99999999999 abc çdelade. $7654321 \mathrm{ab}$ a3 acdefghj 44444.5555555 defghay. Kldefgh yzlk defghjmnp zhlmn nrkmrdn; nmn nyzn ry 12356azynm eeee. Nyzvsx 33333 bcde zındyazl aymçcı. Nkld $11 \mathrm{z}$ snkmorp klmnv abc nrpsştavs. X çrk zmnınrpk aiijklmı. İNTERNETE 4444444, mklnde untrpslmzx habtzlzztab xv ulkmlkgs kmnlm2346, ktaşlpmnr ozxwms, srkmnxy nsp taeirpzxy. Rslxy çynmlxz; tsnmnlkxz çzlmmnvk; pnmȩden ypnkmxzsş. Bx aakmş nd ynxz klmnvş̧ uitkşktlmxz.

Anklmdtsz yzlkntmx km mtknğ grj yntmlaiseixz:

1.Xxx nrdxyzf eeeeeee?

2. Xxx nrdxyf eeeee?

3. Xxxxxx 22222 huikm drlgkmnxy 33333444 ?

4. 8888 hujkm 3333333 YAPIYOR?

5. Hujkm yzlkon nrkmrdnjkn?

6. Snmrı hujkm 3333333666666 ? 
7. Bx aakms ynxz nx 6666666 ?

S.8. Xxx nsalı ibr örğendciıx? Mtindexz yxmnjklsgrty gedrfvbnyu?

S.9. Bgymnhdw olytrdxz lk crthjklş öcvfedsawyu.

S.10. Onut çklt zhjklşpı nk akjytr? Bn çskukmsdy iloy nhuyt yokjhgf?

\%90'I ŞIFRELENMIŞ METIN (Ek 4)

Xxx yyyyyy zzzzzzz vvvvvvv BíNíP ddddd etf eeeee. Kkkkkk ANAHTARIYLA aaaaa bbbb ccccccc çççç. Ççççççççç omferhjke akmn vstjoyrt kyrtd. Pskyjsynf jjjjjjj mmmm. AYAKKABILARINI jjjjjjj öööööööööööö ppppp. Rrrrrrrrr gggg ssssssss şşşşş ttttt rrrrrrr wwwww.

11111222233333333 VARDI. 5555556666666 777777777777. ÖNCE 99999999999 abc çdelade. 7654321ab a3 acdefghj 44444. 5555555 UĞRAŞTI. Kldefgh yzlk defghjmnp zhlmn nrkmrdn; nmn nyzn DA 12356azynm eeee. Nyzvsx 33333 bcde zındyazl aymçcı. Nkld 1lz snkmorp klmnv abc nrpsştavs. X ÇOK zmnınrpk aiijklmı. iNTERNETE 4444444, mklnde untrpslmzx habtzlzztab xv ulkmlkgs kmnlm2346, ktaşlpmnr ozxwms, srkmnxy NOT taeirpzxy. Rslxy çynmlxz; tsnmnlkxz çzlmmnvk; pnmçden ypnkmxzsş. BU aakmş nd ynxz klmnvsş uitkşktlmxz.

Anklmdtsz yzlkntmx km mtknğ GÖRE yntmla1seıxz:

1.Xxx nrdxyzf eeeeeee?

2. Xxx nrdxyf eeeeee?

3. Xxxxxx 22222 HANGİ drlgkmnxy 33333444 ?

4. 8888 hujkm 3333333 YAPIYOR?

5. Hujkm yzlkon ZORLANIYOR?

6. Snmr1 hujkm 3333333666666 ?

7. Bx aakms ynxz nx 6666666 ?

S.8. Xxx nsalı ibr örğendciıx? METINDEN yxmnjklsgrty gedrfvbnyu?

S.9. Bgymnhdw olytrdxz lk crthjklş öcvfedsawyu.

S.10. Onut çklt zhjklşpı nk akjytr? Bn çskukmsdy iloy NELER yokjhgf?

\%80'İ ŞİFRELENMİ̧ METÍN (Ek 5)

Xxx yyyyyy ÇIKINCA, vvvvvvv BiNiP ddddd etf eeeee. Kkkkkk ANAHTARIYLA aaaaa AÇIP cceccec ççççç. Ççççççççç omferhjke akmn vstjoyrt kyrtd. Pskyjsynf jjjjjjj ASTI. AYAKKABILARINI jjjjjjj öööööööööööö ppppp. ÇANTASINI gggg sssssssss şşşşş ttttt rrrrrrr wwwww.

YARIN 222233333333 VARDI. ONLARI 666666677777777777 . ÖNCE 99999999999 abc çdelade. ÇÖZÜLECEK a3 acdefghj 44444. 5555555 
UĞRAŞTI. Kldefgh yzlk KENDİSiNi zhlmn nrkmrdn; AMA nyzn DA 12356azynm eeee. Nyzvsx 33333 bcde zındyazl aymçcı. Nkld ılz snkmorp klmnv abc nrpsştavs. X ÇOK zmnınrpk aiijklmı. iNTERNETE 4444444, mklnde untrpslmzx habtzlzztab xv ulkmlkgs kmnlm2346, KITAPLARI ozxwms, srkmnxy NOT taeirpzxy. Rslxy çynmlxz; tsnmnlkxz çzlmmnvk; PLANLAR ypnkmxzsş. BU aakmş nd ynxz klmnvsş uttkşktlmxz.

AŞAĞIDAKİ yzlkntmx BU mtknğ GÖRE yntmlaıseıxz:

1.Xxx NEREDEN eeeeee?

2. Xxx nrdxyf eeeee?

3. Xxxxxx 22222 HANGİ drlgkmnxy 33333444 ?

4. 8888 hujkm 3333333 YAPIYOR?

5. Hujkm yzlkon ZORLANIYOR?

6. Snmrı HANGİ 3333333 666666?

7. Bx aakms ynxz NE 6666666 ?

S.8. Xxx nsalı ibr örğendcix? METíNDEN yxmnjklsgrty gedrfvbnyu?

S.9. Bgymnhdw olytrdxz ÜÇ crthjklş öcvfedsawyu.

S.10. Onut çklt zhjklşpı nk akjytr? Bn çskukmsdy iloy NELER yokjhgf?

\%70'i ŞIFRELENMIŞ METÍN (Ek 6)

ALI yyyyyy ÇIKINCA, vvvvvvv BíNiP ddddd etf eeee. KAPIYI ANAHTARIYLA aaaaa AÇIP ICCERRIYE ççççc. Ççççççççç omferhjke akmn vstjoyrt kyrtd. Pskyjsynf ÇIKARIP ASTI. AYAKKABILARINI ÇIKARIP öööööööööööö ppppp. ÇANTASINI ALIP ssssssss şşşş̧ ttttt rrrrrrr wwwww.

YARIN İÇIN 33333333 VARDI. ONLARI 6666666 GEREKIYYRDU. ÖNCE 99999999999 abc çdelade. ÇÖZÜLECEK a3 acdefghj VARDI. 5555555 UĞRAŞTI. Kldefgh yzlk KENDİSİNi zhlmn ZORLADI; AMA nyzn DA 12356azynm GELDİ. Nyzvsx 33333 bede zındyazl aymçcı. Nkld ılz snkmorp klmnv abc nrpsştavs. X ÇOK zmnınrpk arijklmı. iNTERNETE 4444444, mklnde untrpslmzx habtzlzztab xv ulkmlkgs kmnlm2346, KíTAPLARI ozxwms, srkmnxy NOT taeirpzxy. Rslxy çynmlxz; tsnmnlkxz çzlmmnvk; PLANLAR ypnkmxzsş. BU aakmş nd ynxz klmnvsş UĞRAŞACAKTI

AŞAĞIDAKİ yzlkntmx BU mtknğ GÖRE yntmlaıseıxz:

1. ALİ NEREDEN eeeeee?

2. ALİ nrdxyf eeeee?

3. ALI' 'NíN 22222 HANGİ drlgkmnxy 33333444 ?

4. 8888 hujkm 3333333 YAPIYOR? 
5. Hujkm yzlkon ZORLANIYOR?

6. Snmrı HANGİ 3333333666666 ?

7. Bx aakms ynxz NE 6666666?

S.8. ALİ nsalı ibr örğendciıx? METíNDEN yxmnjklsgrty gedrfvbnyu?

S.9. Bgymnhdw olytrdxz ÜÇ crthjklş öcvfedsawyu.

S.10. ONUN çklt zhjklşpı nk akjytr? Bn çskukmsdy iloy NELER yokjhgf?

\%60'I ŞIFRELENMIŞ METIN (Ek 7)

ALİ yyyyyy ÇIKINCA, SERVISE BİNiP ddddd etf eeee. KAPIYI ANAHTARIYLA YAVAŞÇA AÇIP ICÇERIYYE GİRDİ. Ççççççççç omferhjke akmn vstjoyrt kyrtd. Pskyjsynf ÇIKARIP ASTI. AYAKKABILARINI ÇIKARIP öööööööööööö ppppp. ÇANTASINI ALIP ssssssss şşşş̧ ttttt rrrrrrr GITTí.

YARIN İÇIN ÖDEVLERİ VARDI. ONLARI 6666666 GEREKIYYORDU. ÖNCE 99999999999 İŞE çdelade. ÇÖZÜLECEK a3 acdefghj VARDI. 5555555 UĞRAŞTI. Kldefgh yzlk KENDISIINI BİRAZ ZORLADI; AMA nyzn DA 12356azynm GELDİ. Nyzvsx 33333 UZUN zındyazl aymçcı. ASIL 1lz snkmorp klmnv İLE nrpsştavs. O ÇOK zmnınrpk aiijklmı. iNTERNETE 4444444, İLGILII untrpslmzx habtzlzztab VE ulkmlkgs kmnlm2346, KiTAPLARI ozxwms, srkmnxy NOT taeirpzxy. Rslxy çynmlxz; tsnmnlkxz çzlmmnvk; PLANLAR ypnkmxzsş. BU aakmş nd ynxz klmnvş̧ UĞRAŞACAKTI

AŞAĞIDAKİ yzlkntmx BU mtknğ GÖRE yntmlaıseıxz:

1. ALİ NEREDEN eeeeee?

2. ALİ nrdxyf eeeee?

3. ALI'NIIN 22222 HANGI drlgkmnxy 33333 444?

4. ÖNCE hujkm 3333333 YAPIYOR?

5. HANGİ yzlkon ZORLANIYOR?

6. Snmrı HANGİ 3333333666666 ?

7. BU aakms ynxz NE 6666666?

S.8. ALİ nsalı ibr örğendciıx? METÍNDEN yxmnjklsgrty gedrfvbnyu?

S.9. BURADAKİ olytrdxz ÜÇ crthjklş öcvfedsawyu.

S.10. ONUN çklt zhjklşpı nk akjytr? Bn çskukmsdy iloy NELER yokjhgf?

\%50'Si ŞiFRELENMİŞ METIN (Ek 8)

ALI yyyyyy ÇIKINCA, SERVISE BİNIP ddddd etf eeee. KAPIYI ANAHTARIYLA YAVAŞÇA AÇIP IÇERIYYE GIRDİ. ÇANTASINI omferhjke 
akmn vstjoyrt kyrtd. Pskyjsynf ÇIKARIP ASTI. AYAKKABILARINI ÇIKARIP öööööööööööö GIYDDI. ÇANTASINI ALIP ssssssss ttttt GEÇiP rrrrrrr GITTTI.

YARIN ICÇIN ÖDEVLERİ VARDI. ONLARI 6666666 GEREKIYYORDU. ÖNCE 99999999999 İŞE çdelade. ÇÖZÜLECEK a3 acdefghj VARDI. 5555555 UĞRAŞTI. Kldefgh yzlk KENDÍSİNi BİRAZ ZORLADI; AMA nyzn DA 12356azynm GELDİ. Nyzvsx 33333 UZUN zındyazl aymçcı. ASIL 1lz snkmorp klmnv İLE nrpsştavs. O ÇOK zmnınrpk aiijklmı. iNTERNETE 4444444, İLGILİ untrpslmzx habtzlzztab VE ulkmlkgs kmnlm2346, KİTAPLARI ozxwms, srkmnxy NOT taeirpzxy. Rslxy çynmlxz; tsnmnlkxz çzlmmnvk; PLANLAR ypnkmxzsş. BU aakmş nd ynxz klmnvsş UĞRAŞACAKTI

AŞAĞIDAKİ yzlkntmx BU mtknğ GÖRE yntmlaıseıxz:

1. ALİ NEREDEN eeeeeee?

2. ALI NEREYE eeeee?

3. ALI'NIIN 22222 HANGİ drlgkmnxy 33333 VAR?

4. ÖNCE hujkm 3333333 YAPIYOR?

5. HANGİ yzlkon ZORLANIYOR?

6. SONRA HANGİ 3333333 YAPIYOR?

7. BU aakms YINE NE YAPIYOR?

S.8. ALİ nsalı BİR örğendciı? METINDEN yxmnjklsgrty SÖYLEYINIZZ?

S.9. BURADAKİ OLAYLARI ÜÇ CÜMLEYLE öcvfedsawyu.

S.10. ONUN çklt zhjklşpı NE akjytr? BUNUN iloy NELER yokjhgf?

\%40'I ŞIFRELENMIŞ METIN (Ek 9)

ALİ yyyyyy ÇIKINCA, SERVISE BİNIP HEMEN etf eeee. KAPIYI ANAHTARIYLA YAVAŞÇA AÇIP IÇERIYE GİRDI. ÇANTASINI omferhjke akmn vstjoyrt kyrtd. Pskyjsynf ÇIKARIP ASTI. AYAKKABILARINI ÇIKARIP TERLIKLERINII GIYDİ. ÇANTASINI ALIP KORIDORU tttt GEÇIP rrrrrrr GITTI.

YARIN ICÇIN ÖDEVLERİ VARDI. ONLARI YAPMASI GEREKIYYORDU. ÖNCE 99999999999 İŞE çdelade. ÇÖZÜLECEK a3 acdefghj VARDI. ONLARLA UĞRAŞTI. Kldefgh yzlk KENDİSINII BİRAZ ZORLADI; AMA ONUN DA 12356azynm GELDİ. Nyzvsx 33333 UZUN zındyazl ALMADI. ASIL İşì snkmorp klmnv İLE nrpsştavs. O ÇOK zmnınrpk aijklmı. iNTERNETE 4444444, İLGíLi untrpslmzx habtzlzztab VE ulkmlkgs KONUŞUYOR, KITAPLARI OKUYOR, srkmnxy NOT taeirpzxy. Rslxy çynmlxz; tsnmnlkxz çzlmmnvk; PLANLAR ypnkmxzsş. BU aakmş nd ynxz klmnvsş UĞRAŞACAKTI

AŞAĞIDAKİ yzlkntmx BU mtknğ GÖRE yntmlaıseıxz: 
1. ALI NEREDEN eeeeee?

2. ALİ NEREYE eeeeee?

3. ALI'’NİN YARIN HANGİ drlgkmnxy ÖDEVİ VAR?

4. ÖNCE hujkm ÖDEVINI YAPIYOR?

5. HANGI yzlkon ZORLANIYOR?

6. SONRA HANGI ÖDEVINI YAPIYOR?

7. BU aakms YINE NE YAPIYOR?

S.8. ALİ nsalı BİR örğendcix? METINDEN yxmnjklsgrty SÖYLEYINi̇Z?

S.9. BURADAKİ OLAYLARI ÜÇ CÜMLEYLE öcvfedsawyu.

S.10. ONUN ÇOĞU zhjklşpı NE ALIYOR? BUNUN İÇíN NELER yokjhgf?

\%30’U ŞIFRELENMIŞ METIN (Ek 10)

ALİ yyyyyy ÇIKINCA, SERVISE BINIP HEMEN etf eeee. KAPIYI ANAHTARIYLA YAVAŞÇA AÇIP İÇERIYE GIRDİ. ÇANTASINI OMUZUNDAN ALIP vstjoyrt KOYDU. Pskyjsynf ÇIKARIP ASTI. AYAKKABILARINI ÇIKARIP TERLIKLERINI GIYDİ. ÇANTASINI ALIP KORIDORU HIZLA GEÇIP ODASINA GITTI.

YARIN ICÇIN ÖDEVLERİ VARDI. ONLARI YAPMASI GEREKIYORDU. ÖNCE 99999999999 İŞE BAŞLADI. ÇÖZÜLECEK a3 acdefghj VARDI. ONLARLA UĞRAŞTI. Kldefgh yzlk KENDİSINİ BİRAZ ZORLADI; AMA ONUN DA ÜSTESINNDEN GELDİ. NyzvSX 33333 UZUN ZAMANINI ALMADI. ASIL İşi SEÇTIĞí klmnv İLE İLGILIYYDi. O ÇOK zmnınrpk aijklmı. iNTERNETE 4444444, İLGÍLİ untrpslmzx habtzlzztab VE ulkmlkgs KONUŞUYOR, KITAPLARI OKUYOR, srkmnxy NOT taeirpzxy. Rslxy çynmlxz; tsnmnlkxz çzlmmnvk; PLANLAR ypnkmxzsş. BU aakmş nd ynxz klmnvş̧ UĞRAŞACAKTI

AŞAĞIDAKİ SORULARI BU mtknğ GÖRE YANITLAYINIZ:

1. ALİ NEREDEN eeeeee?

2. ALİ NEREYE eeeee?

3. ALI'NIIN YARIN HANGİ drlgkmnxy ÖDEVİ VAR?

4. ÖNCE hujkm ÖDEVINI Y YAPIYOR?

5. HANGİ yzlkon ZORLANIYOR?

6. SONRA HANGI ÖDEVINI YAPIYOR?

7. BU AKŞAM YINE NE YAPIYOR? 
S.8. ALİ NASIL BİR örğendciix? METINDEN yxmnjklsgrty SÖYLEYINIZ?

S.9. BURADAKI OLAYLARI ÜÇ CÜMLEYLE ÖZETLEYINIZ.

S.10. ONUN ÇOĞU ZAMANINI NE ALIYOR? BUNUN ICÇIN NELER yokjhgf?

\%20'SI şiFRELENMIŞ METIN (Ek 11)

ALİ yyyyyy ÇIKINCA, SERVISE BINIP HEMEN etf GELDİ. KAPIYI ANAHTARIYLA YAVAŞÇA AÇIP İÇERIYE GİRDİ. ÇANTASINI OMUZUNDAN ALIP VESTIYERE KOYDU. PALTOSUNU ÇIKARIP ASTI. AYAKKABILARINI ÇIKARIP TERLIKLERINİ GIYDII. ÇANTASINI ALIP KORIDORU HIZLA GEÇIP ODASINA GITTİ.

YARIN IÇIIN ÖDEVLERI VARDI. ONLARI YAPMASI GEREKIYORDU. ÖNCE 99999999999 İŞE BAŞLADI. ÇÖZÜLECEK ON acdefghj VARDI. ONLARLA UĞRAŞTI. BEŞINCİ yzlk KENDISININI BİRAZ ZORLADI; AMA ONUN DA ÜSTESINDEN GELDI. NyZvSX ÖDEVI UZUN ZAMANINI ALMADI. ASIL İşi SEÇTIĞİ klmnv İLE İLGILIYYDi. O ÇOK ZAMANINI aijklmı. iNTERNETE GIRIYOR, ILGGILI untrpslmzx habtzlzztab VE UZMANLARLA KONUŞUYOR, KITAPLARI OKUYOR, srkmnxy NOT TUTUYORDU. Rslxy çynmlxz; tsnmnlkxz çzlmmnvk;PLANLAR ypnkmxzsş. BU aakmş nd ynxz klmnvş̧ UĞRAŞACAKTI

AŞAĞIDAKI SORULARI BU METNE GÖRE YANITLAYINIZ:

1. ALİ NEREDEN GELIYOR?

2. ALI NEREYE GELIYOR?

3. ALI'NIIN YARIN HANGİ drlgkmnxy ÖDEVİ VAR?

4. ÖNCE HANGI ÖDEVINI YAPIYOR?

5. HANGI SORUDA ZORLANIYOR?

6. SONRA HANGI ÖDEVINI YAPIYOR?

7. BU AKŞAM YINE NE YAPIYOR?

S.8. ALİ NASIL BİR ÖĞRENCİIIR? METINDEN yxmnjklsgrty SÖYLEYINIZ?

S.9. BURADAKİ OLAYLARI ÜÇ CÜMLEYLE ÖZETLEYINİZ. yokjhgf?

S.10. ONUN ÇOĞU ZAMANINI NE ALIYOR? BUNUN IÇCiN NELER

\%10'U ŞIFRELENMIŞ METIN (Ek 12)

ALI OKULDAN ÇIKINCA, SERVISE BINIIP HEMEN EVE GELDİ. KAPIYI ANAHTARIYLA YAVAŞÇA AÇIP IÇERIYE GIRDİ. ÇANTASINI 
OMUZUNDAN ALIP VESTIYERE KOYDU. PALTOSUNU ÇIKARIP ASTI. AYAKKABILARINI ÇIKARIP TERLIKLERINII GIYDİ. ÇANTASINI ALIP KORIDORU HIZLA GEÇIP ODASINA GITTI.

YARIN IÇIIN ÖDEVLERİ VARDI. ONLARI YAPMASI GEREKIYYORDU. ÖNCE MATEMATIKLE ISŞE BAŞLADI. ÇÖZÜLECEK ON PROBLEM VARDI. ONLARLA UĞRAŞTI. BEŞINACI SORU KENDISISINI BİRAZ ZORLADI; AMA ONUN DA ÜSTESINDEN GELDI. TÜRKÇE ÖDEVI UZUN ZAMANINI ALMADI. ASIL İşi SEÇTIĞİ klmnv İLE İLGÍLIYYII. O ÇOK ZAMANINI ALIYORDU. iNTERNETE GIRIYYOR, ILGILI untrpsImzx habtzlzztab VE UZMANLARLA KONUŞUYOR, KITAPLARI OKUYOR, srkmnxy NOT TUTUYORDU. Rslxy çynmlxz; tsnmnlkxz çzlmmnvk; PLANLAR ypnkmxzsş. BU AKŞAM DA YİNE klmnvsş UĞRAŞACAKTI

AŞAĞIDAKİ SORULARI BU METNE GÖRE YANITLAYINIZ:

1. ALİ NEREDEN GELIYYOR?

2. ALİ NEREYE GELIYOR?

3. ALI'NIN YARIN HANGİ DERSLERDEN ÖDEVİ VAR?

4. ÖNCE HANGI ÖDEVINİ YAPIYOR?

5. HANGI SORUDA ZORLANIYOR?

6. SONRA HANGI ÖDEVINI YAPIYOR?

7. BU AKŞAM YİNE NE YAPIYOR? SÖYLEYINIZ?

S.8. ALİ NASIL BİR ÖĞRENCİDİR? METINDEN yxmnjklsgrty

S.9. BURADAKİ OLAYLARI ÜÇ CÜMLEYLE ÖZETLEYINİZ.

S.10. ONUN ÇOĞU ZAMANINI NE ALIYOR? BUNUN IÇIIN NELER YAPIYOR?

\%01'I ŞİFRELENMIŞ METIN (Ek 13)

ALİ OKULDAN ÇIKINCA, SERVISE BINIIP HEMEN EVE GELDI. KAPIYI ANAHTARIYLA YAVAŞÇA AÇIP IÇERIYY GIRDİ. ÇANTASINI OMUZUNDAN ALIP VESTIYERE KOYDU. PALTOSUNU ÇIKARIP ASTI. AYAKKABILARINI ÇIKARIP TERLIKLERINI GIYDII. ÇANTASINI ALIP KORIDORU HIZLA GEÇIP ODASINA GITTI.

YARIN IÇIN ÖDEVLERİ VARDI. ONLARI YAPMASI GEREKIYYRDU. ÖNCE MATEMATIKLE İŞE BAŞLADI. ÇÖZÜLECEK ON PROBLEM VARDI. ONLARLA UĞRAŞTI. BEŞINCI SORU KENDISIINI BIIRAZ ZORLADI; AMA ONUN DA ÜSTESINDEN GELDİ. TÜRKÇE ÖDEVİ UZUN ZAMANINI ALMADI. ASIL İşi SEÇTÍĞİ klmnv İLE İLGILIYYII. O ÇOK ZAMANINI ALIYORDU. iNTERNETE GİRIYOR, 
ÍLGILI ÜNIVERSITE HOCALARIYLA VE UZMANL ÇE ARLA KONUŞUYOR, KITAPLARI OKUYOR, SÜREKLİ NOT TUTUYORDU. RESIM ÇEKIYYOR; TASLAKLAR ÇIZIYYOR; PLANLAR YAPIYORDU. BU AKŞAM DA YİNE klmnvş̧ UĞRAŞACAKTI

AŞAĞIDAKI SORULARI BU METNE GÖRE YANITLAYINIZ:

1. ALİ NEREDEN GELIYOR?

2. ALİ NEREYE GELIYOR?

3. ALI'NIN YARIN HANGİ DERSLERDEN ÖDEVI VAR?

4. ÖNCE HANGI ÖDEVINI YAPIYOR?

5. HANGI SORUDA ZORLANIYOR?

6. SONRA HANGİ ÖDEVINİ YAPIYOR?

7. BU AKŞAM YINE NE YAPIYOR?

S.8. ALİ NASIL BİR ÖĞRENCIDIIR? METINDEN YARARLANARAK SÖYLEYINIZ?

S.9. BURADAKI OLAYLARI ÜÇ CÜMLEYLE ÖZETLEYINIZ.

S.10. ONUN ÇOĞU ZAMANINI NE ALIYOR? BUNUN IÇIN NELER YAPIYOR? 